\title{
The Visualisation of Web Usage
}

\author{
R. Spence ${ }^{1}$, S. Chatty ${ }^{2}$, H. Christensen ${ }^{3}$, K. Fishkin ${ }^{4}$, L. Johnston ${ }^{5}$, N. de

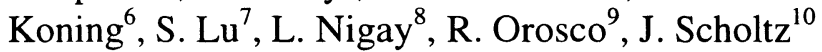 \\ 1 Imperial College, London \\ 2 CENA, France \\ 3 University of Aarhus, Denmark \\ 4 Xerox PARC, USA \\ 5 University of Melbourne, Australia \\ 6 Baan Labs, The Netherlands \\ 7 CSIRO, Australia \\ 8 CLIPS-IMAG, University of Grenoble, France \\ 9 University Autonoma de Madrid, Spain \\ 10 NIST, USA
}

Abstract: In the course of a six-hour workshop, participants from a wide range of backgrounds considered the task of designing a visualisation tool on behalf of an investigator interested in the behaviour exhibited by users of a web site. The group identified the motivation behind the search for a tool as well as examples of the type of question the tool might have to answer. It then proposed a variety of potentially useful visualisation tools, techniques and concepts, and carried out a first evaluation, partly assisted by reference to an aide-memoire which had resulted from an earlier brainstorming session. The result was deemed to be useful for transmission to the problem-holder to act as a starting point for more intensive study, prototyping and evaluation.

Key words: Visualisation techniques, World Wide Web, Design Method.

The original version of this chapter was revised: The copyright line was incorrect. This has been corrected. The Erratum to this chapter is available at DOI: 10.1007/978-0-387-35349-4_22 


\section{THE SCENARIO}

\subsection{Motivation}

The scenario selected for attention was the acquisition of insight into the behaviour of users of a web site. There are many motivations for acquiring such insight, especially if the site has commercial relevance. For example: to provide a more comprehensive interpretation of large amounts of data than more traditional interpretations of separate metrics; to allow exploration of data to see possible trends, perhaps by filtering data and grouping nodes; to facilitate comparisons of usage before and after a modification; to see the effect of different browsers and different cultural backgrounds; to examine an overview of use patterns, thereby helping to focus detailed user studies (for example on parts of sites not used, and short loops); and generally to compare expected use of the site with actual use to see if design objectives are being met. Particularly for the designer of a commercial web site (here, 'site' can be one page or a grouping of pages), it may be desirable for users to quickly find the data they seek, and to spend a reasonable time there. Alternatively, it might be desirable to allow users to find what they want quickly, but then to have a variety of tempting items on the way out. Sites that serve as reference or informational sites may profitably support rapid entrance and exit.

Current web usage statistics focus on hits per page, which has little to do with user paths. If a designer constructs a web site based on a model, then being able to view usage to see if it conforms with the model is essential.

\subsection{Questions}

A first evaluation of any proposed visualisation tool or technique benefits from a list of questions an investigator might pose. The list included:

- Are people entering my site as I thought?

- Are there de facto front pages?

- If I have 'goal' pages, how can I characterise users who do not get there?

- Are there 'poison' pages that scare people away from further exploration?

- Is a given user visiting a given node more than once per session (this could be desirable, or not)?

- How are people getting to my site? (referral from another site? web search engine? bookmark?)

- Are people coming to my site by mistake?

- Where do people go afterwards?

- What is the average path length to a node?

- How many nodes are visited per session?

- Does my search facility work? 
Design of a visualisation tool should also acknowledge the possibility of questions that cannot be anticipated beforehand. As Cleveland (1985) has pointed out:

"Graphing data needs to be iterative because we often do not know what to expect of the data; a graph can help discover unknown aspects of the data, and once the unknown is known, we frequently find ourselves formulating new questions about the data" (our italics)

\subsection{A Model}

A natural model for discussion is the node-link directed graph (Figure 1). It reflects the essential connectivity of the Web while supporting consideration not only of the numeric aspects of Web usage (for example, the number of times a node is visited) but also temporal behaviour. As shown, it may be useful to indicate notional entry and exit points.

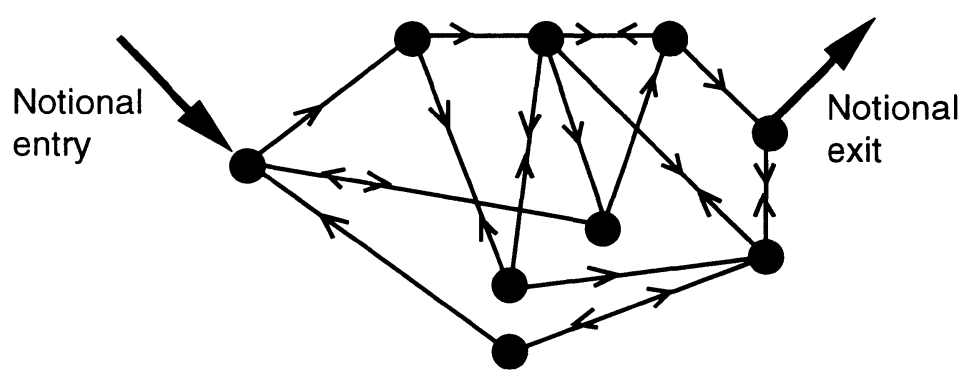

Figure 1. Node-Link representation of a web site

\section{REPRESENTATIONS}

The selection of a representation for Web behaviour must bear in mind the meaning of visualisation:

"the formation of an internal mental model"

Visualisation is something a human being - here, the investigator - does, by viewing and interacting with some externalisation (i.e., display) of the data. The overall goal of the investigator is to be able to perform various tasks, supported by an internal mental model derived via a visualisation tool. Consequently we need to evaluate the various representations of web usage according to those tasks. To identify the tasks the list of questions of section 1.2 is used as a starting point. 


\section{$2.1 \quad$ Node-Link representation}

It is reasonable to enquire whether the node-link model (Figure 1) provides a suitable representational basis. It was suggested that a disadvantage would be associated with the high data density, leading to a confusing display, though some amelioration may be obtained by means of distortion ('fisheye') techniques (see section 3). It was thought there would be some difficulty in grouping nodes, and probably some difficulty in interpreting temporal behaviour. A node-link diagram representing a Web site does not have the same spatial significance that is associated with the representation of many physical networks. Nevertheless, potentially it was thought to be worth investigating transformations such as those associated with node-link and node-loop duality (Seshu \& Reed, 1961).

\subsection{Landscape representation}

Established work by Chalmers $(1992,1993)$ and others has illustrated the $2.5 \mathrm{D}$ 'landscape' metaphor, sometimes in the context of showing the extent of a relationship between a collection of items. In Chalmer's BEAD system, items in a database (the papers in two conferences) were compared on the basis of a selected set of keywords, and their location in multi-dimensional space determined by their overall similarity. A mathematical projection of that data into $2.5 \mathrm{D}$ space leads to a landscape presentation in which similar items are seen to be clustered together.
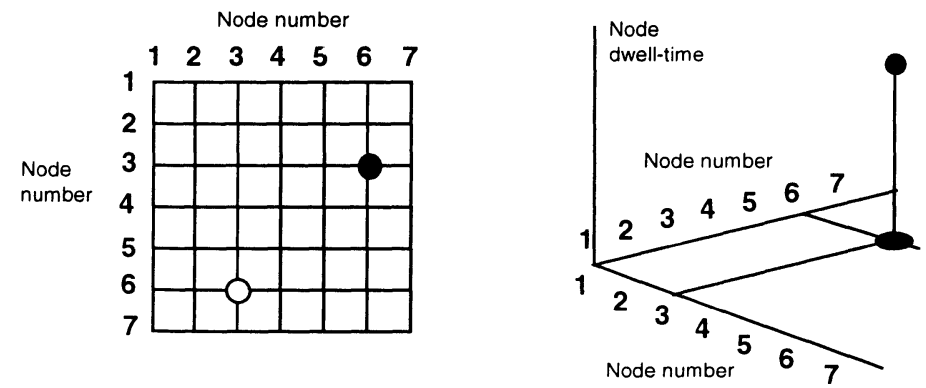

Figure 2. Node-to-node transition Figure 3. Node dwell-time following a transition

A possibly novel landscape representation of Web usage can be introduced by first considering a plane having identical axes which contain the nodes, here represented by integers (Figure 2). A dot $([3,6]$, as shown) can then represent a transition from one node to another, in this case from node 3 to node 6 . The associated point, here [6,3] would represent transition in the reverse direction. Now consider the addition of a third dimension (Figure 3 ) whose value can characterise any of a wide range of Web usage metrics such as node dwell-time. The result will be a number of points in 3-dimensional space that can be joined to form a surface (Figure 4). It is suggested that such a surface might reveal significant aspects of Web usage, particularly if transitions can be reordered interactively and if effective 
use is made of available encoding techniques (e.g., colour) to enrich the display. Exploration using prototypes, following a study of existing literature, will be needed to establish the value of such a landscape representation. The work of Andrews (1995) may well be relevant in this regard.

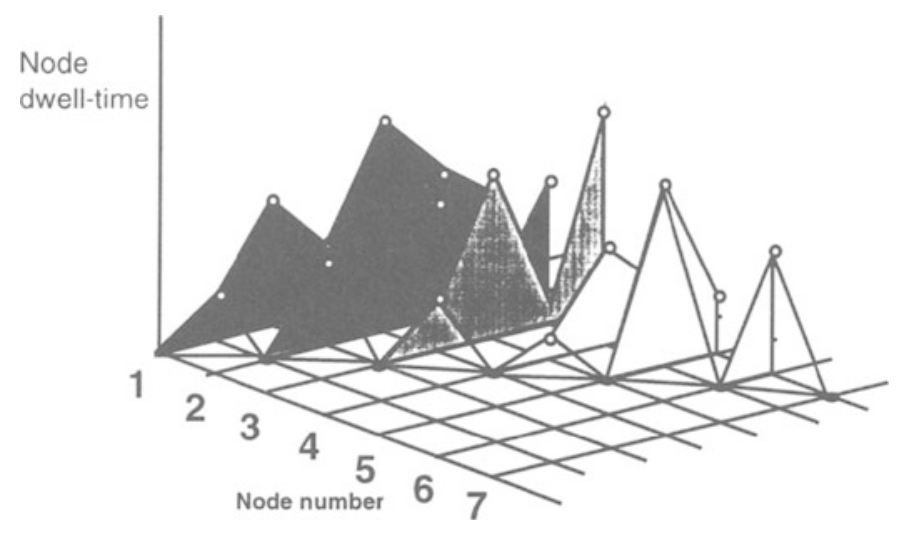

Figure 4. A surface of node dwell-times

\subsection{Netmap ${ }^{\mathrm{rm}}$}

Netmap ${ }^{\text {TM }}$ (Westphal \& Blaxton, 1998) is a proprietary information visualisation tool specifically developed for the study of data principally characterised by connectivity. Its facilities appeared to offer an attractive means for investigating many of the questions (section 1.2) pertinent to Web usage.

In the anticipated use of a product such as Netmap ${ }^{\mathrm{TM}}$, nodes would be associated, possibly in groups sharing some property, with radial segments of an annulus (Figure 5). Within the central circular area, a transition from one node to another - the essential connectivity data - would be represented by a straight line connecting those nodes. The complete history - or even some relatively limited time-slice - of Web usage could well result in such a high density of lines within the central circular area as to significantly impede interpretation. However, thresholding techniques can be employed to suppress lines incident on selected nodes (for example those visited less than $\mathrm{x}$ times) and thereby reveal patterns of usage. Many other enhancements to the underlying concept of Netmap ${ }^{\text {TM }}$ (some already included) can easily be envisaged: they include radial bar charts (Figure 6) representing some node-specific behaviour such as number of visits (a feature offered by the Daisy visualisation tool (Westphal \& Blaxton, 1998)); the clustering, in 'mini-maps', of nodes sharing a common feature; the encoding of lines by thickness and colour; the interactive ordering of groups of nodes; the creation of new segments; and algorithmic support of the kind that may carry out relevant calculations (say of total time through the web site). 
At this stage no firm opinion as to the relative value of various representations could be offered: the further development of ideas and exploration of prototypes is the next step.
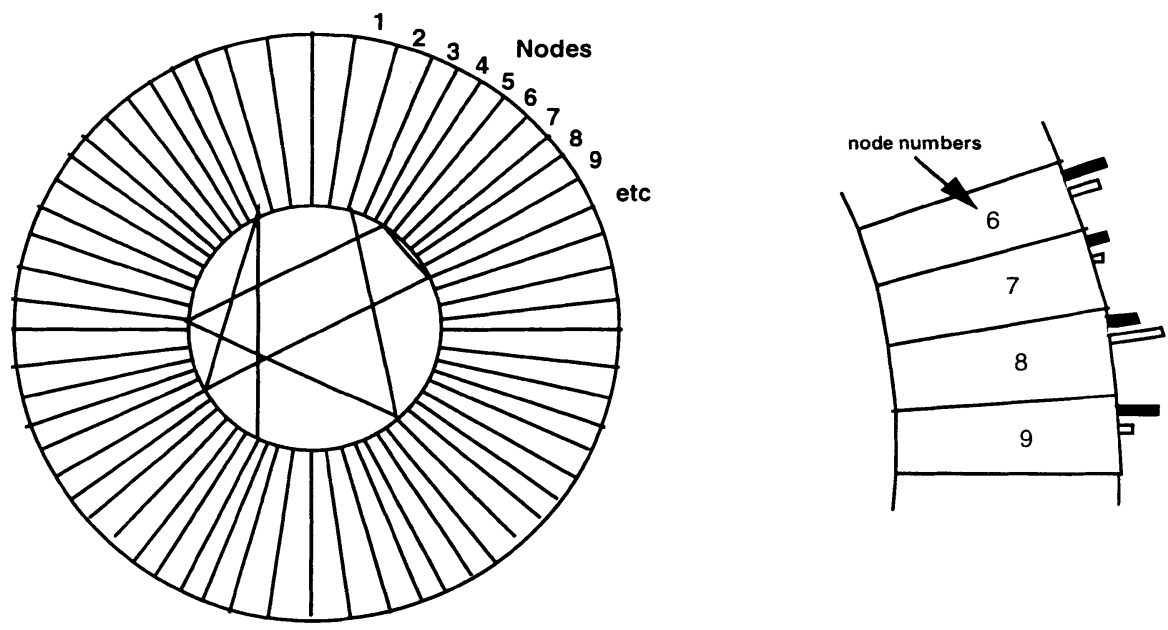

Figure 5. The main Netmap ${ }^{\mathrm{TM}}$ display

Figure 6. Bar charts associated with segments

\subsection{Temporal behaviour}

Temporal aspects of Web usage are of particular interest. The simple 2dimensional representation of Figure 7 may be worth investigation as also might the animation of a Netmap ${ }^{\mathrm{TM}}$-style display and the representation of that display in a third dimension of time. Again, much remains to be discovered about the value of such representations and the way in which a useful visualisation tool might finally emerge. 


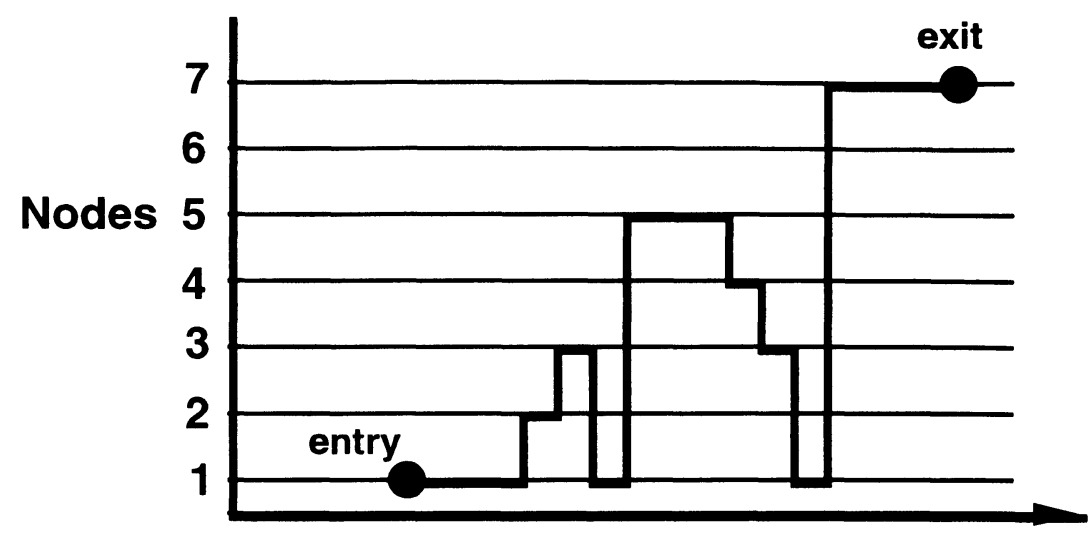

Time

Figure 7. The temporal aspect of node traversal

\section{DESIGN STEPS AND THE QOC TECHNIQUE}

A classical and relevant design approach involving task identification, user interface concept selection and interface definition can benefit from the QOC (Questions, Options, Criteria) technique devised by MacLean et al (1989). Its application to the design of the visualisation tool is briefly illustrated here.

Some of the tasks the investigator will perform can be deduced from the questions of paragraph 1.2: an example is to establish how users reach a particular node.

The next step involves identifying the concepts to be embodied in the interface, an important one being the internal representation of web usage. A possible solution, as remarked in section 2.1, is to base the user interface on the web site's node-link representation (Figure 1): this can afford a direct mapping between the psychological variables of the web site designer and the physical variables manipulated in the user interface, thereby hopefully reducing the designer's cognitive load. 


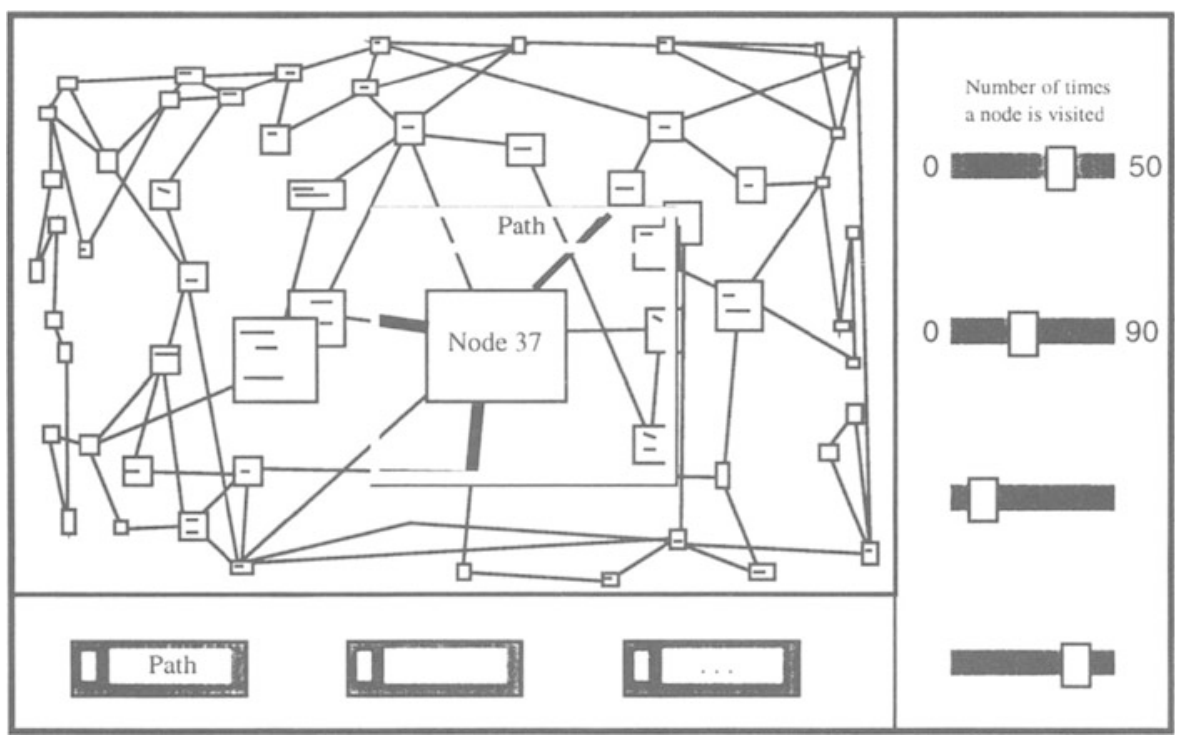

Figure 8. A design solution for the visualisation tool for web usage

The last step is to design the interaction techniques appropriate to the identified task and interface concept. Figure 8 shows one possible design which can be guided by ergonomic requirements using the QOC technique. On the right the palette of alphasliders (Ahlberg, 1994) is dedicated to tasks related to the global graph. On the other hand, each task related to a particular node or link is performed using a Magic Lens (Stone et al, 1994), of which a selection is available under the graph. Finally, the graph itself is displayed in the main part of the window by applying the bifocal/fisheye technique (Spence \& Apperley, 1982; Furnas, 1986). Several views of the graph are possible by filtering it by means of the alphasliders or by investigating a particular node or link using one or more Magic Lens. 


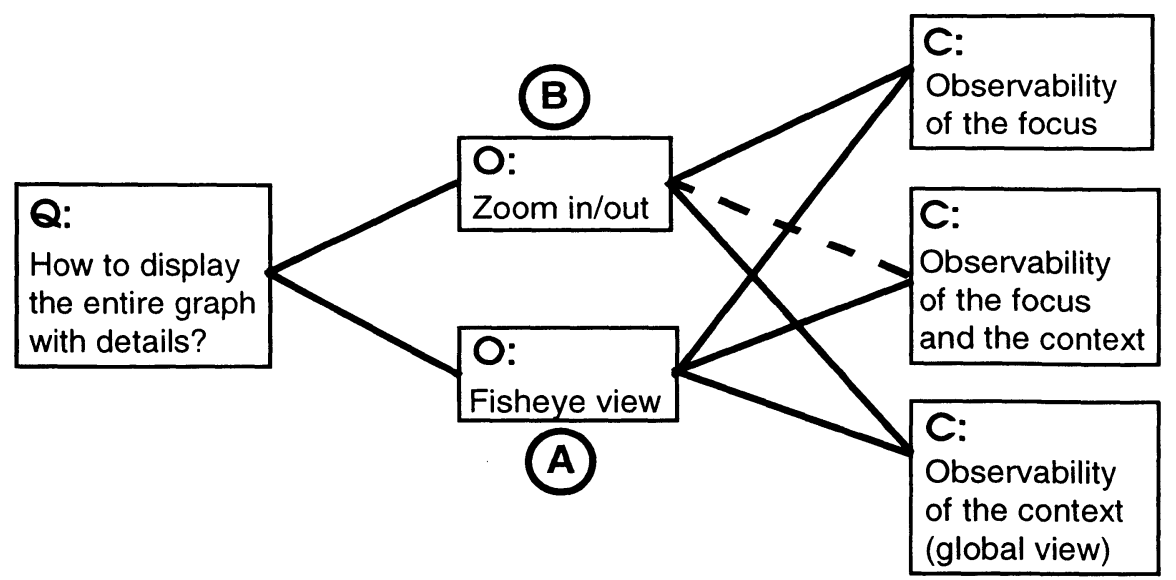

Figure 9. A QOC space to justify the fisheye view of the graph

The QOC technique can be used to rationalise the design in a number of ways. For example, one design issue arises from the need to view the entire graph, but also see enough detail to perform a task. Two options will be examined. In the first (labelled A in the QOC diagram of Figure 9), a subset of the elements of the graph can be observed in detail, while allowing the complete set to be observed without detail, by using 'compression' or 'distortion' procedures, an approach called 'Focus + Context'. The user can rely on global context to orient themselves and on the current focus to make sense of local information. A second option (B) is to provide zoom in/out functions. In this case the investigator can obtain details on demand but then loses global context. In this way the design of Figure 8 is justified using the QOC space shown in Figure 9.

The design solution illustrated in Figure 8 and discussed above may well be acceptable, but only combines already existing visualisation techniques. Nevertheless, under these conditions, the steps appear to provide a framework for design.

\section{CHECKLISTS}

The creation of an effective visualisation tool remains an essentially craft activity: there are no procedures leading directly from a knowledge of data characteristics and the tasks to be performed, to the description of an interactive tool. Moreover, and especially during the past decade, new representations, encoding techniques and interaction mechanisms - as well as new concepts (Tweedie, 1995) are emerging at a rapid rate. The commissioning or design of a new visualisation tool is therefore very much a creative act requiring constant and iterative evaluation. One would therefore expect the person taking the ideas already presented, and 
developing them further, to be reaching for one or other of Tufte's (1990) books for possible inspiration. In fact, the further development of the ideas presented here would benefit from a checklist of some sort. In the limited time available to the authors such a (partial) checklist was generated by a brainstorming technique: following the identification of the representations discussed above, randomly selected items were used as a checklist. One was the concept of filtering by means of the Magic Lens.

\section{CONCLUSION}

The authors felt, after due consideration, that it would be inappropriate to offer a set of guidelines to the person or group given the task of developing the above ideas further, with the eventual intention of implementing an operational visualisation tool. Such a task calls for a considerably expanded study, ranging from the essential motivation and typical questions to a thorough study of available knowledge about information visualisation tools and, eventually, to a great deal of prototype studies and evaluation: the Task/Artefact cycle identified by Carroll (1991) will surely be much in evidence.

\section{REFERENCES}

Ahlberg, C., and Shneiderman, B. (1994) Visual Information Seeking: Tight coupling of dynamic query filters with Starfield displays, ACM, in Proc. CHI'94, pp.313-317.

Andrews, K. (1995) Visualising Cyberspace: Information Visualisation in the Harmony Internet Browser, IEEE, in Proc. Information Visualisation'95, pp.97-104.

Carroll, J., Kellogg, W.A., and Rosson, M.B. (1991) The Task-Artefact Cycle, in Designing Interaction (ed. Carroll), CUP.

Chalmers, M., and Chitson, P. (1992) BEAD: Explorations in Information Visualisation, ACM, in Proc SIGIR, pp.330-337.

Chalmers, M., (1993) Using a Landscape Metaphor to Represent a Corpus of Documents, in Spatial Information Theory (Frank \& Campari, eds), Springer Verlag LNCS, 716, pp.377390.

Cleveland, W.S. (1985) The Elements of Graphing Data, Monterey, Wadsworth.

Furnas, G. (1986) Generalised Fisheye Views, ACM, in Proc. CHI'86, pp.16-23.

MacLean, A., Young, R., and Moran, T. (1989) Design Rationale: the argument behind the artefact, ACM, in Proc. CHI'89, pp.247-252.

Seshu, S., and Reed, M.B. (1961) Linear Graphs and Electrical Networks, Reading, Mass.: Addison-Wesley.

Spence, R., and Apperley, M.D. (1982) Data base navigation: an office environment for the professional, Behaviour and Information Technology, 1, 1, pp.43-54.

Stone, M., Fishkin K., and Bier, E. (1994) The Movable Filter as a User Interface Tool, ACM, in Proc. CHI'94, pp.306-312.

Tufte, E.R. (1990) The Visual Display of Quantitative Information, Graphics Press, Cheshire, Conn. 
Tweedie, L. (1995) Interactive Visualisation Artefacts: how can abstractions inform design? in People and Computers $X$ (eds. Kirby, Dix and Finlay), Cambridge, CUP.

Westphal, C., and Blaxton, T. (1998) Data Mining Solutions, New York, John Wiley.

\section{APPENDIX: AIDE MEMOIRE}

Prior to the consideration of the visualisation of web usage, a brainstorming session generated a large number of terms relevant to visualisation tools. Following the generation of the terms they were assigned to groups, at the time classified as Means, Ends, Requirements, Abstract and Other. Means included Filters and Metaphors; Ends included interpretation time; Requirements included Semantic Zooming and Cognitive Overhead; and Abstract included Derivative Information.

\section{BIOGRAPHY}

Bob Spence is Professor of Information Engineering and Head of the Department of Electrical \& Electronic Engineering at Imperial College, London. His research interests are in the fields of Engineering Design and Human-Computer Interaction. Bob is a Fellow of the Royal Academy of Engineering. 